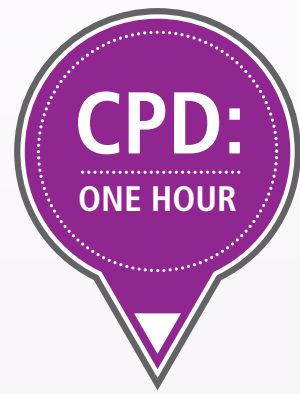
\title{
Sjögren's syndrome
}

Facilitating timely diagnosis of

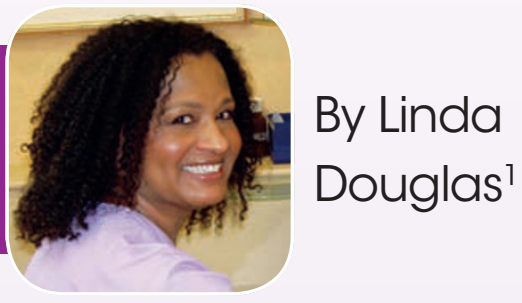

\section{Introduction}

Sjögren's syndrome is an autoimmune disease in which intense lymphocytic infiltration of the exocrine glands ${ }^{1}$ and hyperactivity of $B$ lymphocytes ${ }^{2}$ cause inflammation, which damages glandular tissue and impairs function. Primary Sjögren's syndrome is diagnosed in the absence of any other connective tissue disease, while secondary Sjögren's syndrome is accompanied by other autoimmune diseases, such as rheumatoid arthritis, lupus erythematosus, Graft Versus Host Disease, or in rare cases, Behçet's disease. ${ }^{3}$ Secondary Sjögren's syndrome comprises approximately $60 \%$ of cases.

In January 2012, the Sjögren's Syndrome Foundation launched a 5-year Breakthrough Goal, to reduce the time between onset and diagnosis of Sjögren's by $50 \%$ in five years. When this Goal was launched, research showed the average diagnosis time was nearly six years. ${ }^{4}$

${ }^{7} \mathrm{RDH}$, BSC. Linda M. Douglas graduated as a dental hygienist from the Royal Dental Hospital in London, England in 1982. After graduation she worked in periodontology, before moving to Toronto, Canada, where she has worked in private practice since 1990. Linda also has a BSC in Oral Health Promotion. Linda's desire to support dry mouth patients has instigated an in-depth study of xerostomia, and the related pathologies. She is also Clinical Director for Hygienetown, an online community for dental hygienists, and teaches continuing education courses on xerostomia management, saliva testing, and caring for dental patients with eating disorders.
Research at the University of Toronto found a median delay of four years between onset and diagnosis (range $0-28$ years). ${ }^{5}$ Prompt diagnosis allows access to treatment to relieve symptoms, minimise complications, and enhance vigilance for lymphoma: Sjögren's patients have a 16 times greater risk of developing lymphoma than a healthy individual. ${ }^{6}$

Much of the delay between onset and diagnosis is related to the diverse symptoms, which make diagnosis difficult, plus a lack of awareness of the condition on the part of various healthcare professionals encountered by many Sjögren's patients, who frequently fail to 'connect the dots' regarding the numerous signs and symptoms affecting multiple body systems.

\section{Clinical features of Sjögren's} syndrome

Sjögren's syndrome is mainly characterised neuropathy, ${ }^{7}$ hypothyroidism, and lymphoma.

\section{Sjögren's} syndrome and mental health

In common with many chronic medical diseases, depression or anxiety might accompany Sjögren's syndrome, related to its adverse effect on the quality of life. This is shown by its score on the Devin's Illness Intrusiveness Scale, ${ }^{8}$ where the negative impact on quality of life is comparable with that of multiple sclerosis, or kidney dialysis. ${ }^{9}$ In addition, mounting evidence indicates that chronic exposure to elevated inflammatory cytokines causes persistent alterations in neurotransmitter systems, which can lead to neuropsychiatric disorders and depression. ${ }^{10}$

\section{'ORALRELATED COMPLICATIONS INCLUDE \\ SIALOLITHS, SALIVARY GLAND SWELLING, HIGH \\ CARIES RISK, PERIODONTAL DISEASE, SOFT}

\section{TISSUE INFECTIONS AND ANGULAR CHEILITIS'}

by dry eyes and a dry mouth, and may also affect other mucosal tissues such as the nose, larynx, gastro-intestinal tract and vagina. Dry eyes can lead to blurred vision. Oral-related complications include sialoliths (salivary stones), salivary gland swelling, high caries risk, particularly at the cusp tips and cervical sites, periodontal disease, and soft tissue infections such as candidiasis, and angular cheilitis.

Dysphagia might also occur, which can lead to aspiration pneumonia.

The systemic autoimmunity associated with Sjögren's syndrome can also result in dry skin, fatigue, low grade fever, constipation, myalgia, and joint pain. Other conditions which might occur include small vessel vasculitis, Raynaud's phenomenon, pulmonary symptoms, nephritis,
Prevalence and incidence of Sjögren's syndrome

An estimated 221,583 Britons are affected ${ }^{11}$ and approximately 4 million Americans. Ninety percent of Sjögren's patients are women. ${ }^{12}$ Most are diagnosed in their late 40s, but it can also affect children and males. Males are usually diagnosed later than females.

\section{Diagnosis of Sjögren's syndrome} Accurate diagnosis of Sjögren's syndrome begins with thorough assessment to expedite specialist referral. This includes general appraisal, medical history review, and screening for symptoms using questionnaires, followed by head and neck examination, and comprehensive intra-oral examination. 
Questionnaires to assess symptoms Oral symptoms

A positive response to one or more of the following questions is suggestive of Sjögren's syndrome:

1. Have you had a daily feeling of dry mouth for more than three months?

2. Have you had recurrently or persistently swollen salivary glands as an adult?

3. Do you frequently drink liquids to aid in swallowing dry food?

\section{Ocular symptoms}

A positive response to at least one of the following questions is suggestive of Sjögren's syndrome:

1. Have you had daily, persistent troublesome dry eyes for more than three months?

2. Do you have a recurrent sensation of sand or gravel in the eyes?

3. Do you use tear substitutes more than three times a day?

\begin{tabular}{l|l|l|} 
Table $\mathbf{1}$ Classification criteria for Sjögren's syndrome & Scoring \\
\hline Diagnostic Criteria ltem & 3 points \\
\hline Anti-SSA/Ro antibody positivity & 3 points \\
\hline Focal lymphocytic sialadenitis with a focus score of 1 foci/4 $\mathrm{mm}^{2}$ or greater & 1 point \\
\hline $\begin{array}{l}\text { An abnormal ocular staining score of } 5 \text { or greater (or van Bijsterveld score of } \\
4 \text { or more) }\end{array}$ & 1 point \\
\hline A Schirmer's test result of $5 \mathrm{~mm} / 5 \mathrm{minutes}$ or less & 1 point \\
\hline An unstimulated salivary flow rate of $0.1 \mathrm{ml} / \mathrm{minute}$, or less &
\end{tabular}

established for use in individuals with signs and/ or symptoms suggestive of SS.

These single set classification criteria performed well in validation analyses, demonstrating $96 \%$ sensitivity and $95 \%$ specificity, and are also suitable criteria

\section{'SWELLING OF THE PAROTID AND}

\section{SUBMANDIBULAR SALIVARY GLANDS CAN BE}

\section{REDUCED BY APPLYING HEAT AND MASSAGE}

\section{TO AID SALIVARY FLOW THROUGH THE DUCTS.'}

Diagnostic techniques include blood testing for anti-SSA/Ro antibody, biopsy to assess the minor salivary glands inside the lower lip for focal lymphocytic sialadenitis, ocular staining with Rose Bengal, or the van Bijsterveld score to assess surface damage of the conjunctiva and cornea, Schirmer's test for lachrymal flow, and measurement of unstimulated salivary flow.

Other conditions which cause dry eyes and dry mouth should be considered in differential diagnosis, and excluded. For example: preexisting lymphoma, hepatitis C infection, HIV infection, sarcoidosis, use of anti-cholinergic drugs, or a history of head and neck radiation therapy.

\section{Current diagnostic criteria}

The diagnostic criteria for Sjögren's syndrome is evolving, and has been revised numerous times since 1965.

In 2016, the American College of Rheumatology (ACR) and the European League Against Rheumatism (EULAR) developed and validated an international consensus of data-driven classification criteria for primary Sjögren's syndrome (SS), which were for enrollment in clinical trials. The final classification criteria are based on the weighted sum of five items. ${ }^{13}$

Each objective criterion is allocated points: individuals with signs and/or symptoms suggestive of Sjögren's syndrome and a total score of at least 4 points meet the criteria for diagnosis of primary SS (Table 1).

\section{Management of the Sjögren's} patient in the dental practice setting Extra-oral and intra-oral examination needs to be performed on a regular basis, to assess for complications of Sjögren's syndrome. The Challacombe Scale of Clinical Oral Dryness ${ }^{14}$ (Fig. 1) is a valuable tool for clinically assessing and quantifying the severity of oral dryness, and for evaluating the outcomes of our interventions. This scale is based on a Clinical Oral Dryness Score (CODS), and lists ten key features of dry mouth; one point is allocated for each feature, and the patient's additive score indicates the severity of the dry mouth. There is an inverse relationship between salivary flow rates, and CODS: a high CODS is related to hyposalivation.
Management of the dry mouth needs to be multifaceted, to address the multiple oral symptoms and prevent complications. This includes stimulation of salivary flow, conservation of functional salivary gland tissue, and saliva substitutes as required to protect and lubricate, and facilitate speech, mastication and swallowing. Supplementation with Omega $31,000 \mathrm{mg}$ has been shown to improve the lubricating quality of the saliva, and the tears. ${ }^{15}$

Topical fluoride applications, and calcium and phosphate remineralisation preparations reduce caries risk. ${ }^{16}$ Swelling of the parotid and submandibular salivary glands (Fig. 2) can be reduced by application of heat and massage to aid salivary flow through the ducts.

\section{Conclusion}

We are getting closer to our goal of early diagnosis. In 2016, the Sjögren's Syndrome Foundation annual survey of newly diagnosed patients revealed that for those diagnosed in 2015, it took an average of three years to receive an accurate diagnosis, ${ }^{17}$ compared with almost six years in 2012. This was achieved by increasing public awareness, and increasing education and awareness among healthcare professionals.

Increased awareness of Sjögren's syndrome in the medical and dental communities facilitates improved outcomes for the Sjögren's patient, by enhancing inter-professional collaboration and vigilance. For example: screening questionnaires utilised by both medical and dental professionals could include questions on ocular, oral and systemic symptoms of Sjögren's syndrome.

Raising public awareness with more widely disseminated information on Sjögren's syndrome, and increased availability of screening questionnaires for self-assessment could be valuable. Knowledge also empowers patients to advocate for themselves as they navigate the healthcare system. 


\section{The Challacombe Scale}

The Challacombe Scale was developed from research conducted at King's College London Dental Institute under the supervision of Professor Stephen Challacombe*. The purpose of this scale is to be able to visually identify and quantify whether your patient has xerostomia (dry mouth) and if so, how it changes over time and the most appropriate therapy options. This scale is applicable whatever your profession.

The Challacombe Scale works as an additive score of 1 to $10: 1$ being the least and 10 being the most severe. Each feature scores 1 and symptoms will not necessarily progress in the order shown, but summated scores indicate likely patient needs. Score changes over time can be used to monitor symptom progression or regression.

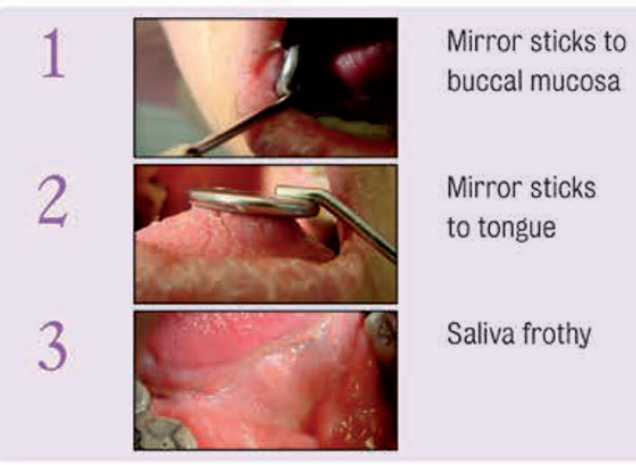

$\begin{array}{lll}\text { No saliva pooling } & \begin{array}{l}\text { An additive score of } 4-6 \text { indicates } \\ \text { in floor of mouth }\end{array} & \begin{array}{l}\text { Tongue shows generalised shortened } \\ \text { mapillae (mild depapillation) } \\ \text { gum or simple sialogogues may be } \\ \text { required. Needs to be investigated } \\ \text { further if reasons for dryness are not } \\ \text { clear. Saliva substitutes and topical } \\ \text { fluoride may be helpful. Monitor at } \\ \text { regular intervals especially for early } \\ \text { decay and symptom change. }\end{array}\end{array}$

An additive score of $1 \cdot 3$ indicates mild dryness. May not need treatment or management. Sugar-free chewing gum for 15 mins, twice daily and attention to hydration is needed. Many drugs will cause mild dryness. Routine checkup monitoring required.

(ie. smooth)

An additive score of $7-10$ indicates severe dryness. Saliva substitutes and topical fluoride usually needed. Cause of hyposalivation needs to be ascertained and Sjögrens Syndrome excluded. Refer for investigation and diagnosis. Patients then need to be monitored for changing symptoms and signs, with possible further specialist input if worsening.

\section{Cervical caries (more than two teeth)}

Debris on palate

or sticking to teeth

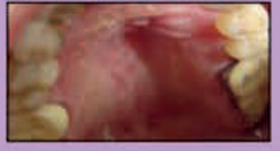

Tongue lobulated/

fissured Free CPD 
1. Sjögren's Society of Canada. What is Sjögren's? Available at: http:// sjogrenscanada.org/ (accessed January 2018).

2. Pers J-O, Youinou P. B Lymphocytes in Primary Sjögren's syndrome. 2016; 217 228. 10.1016/B978-0-12-803604-4.00014-9.

3. Erten S, Erkol E, Perçinel S, Ölmez U, Ensari A, Düzgün N. A case with Sjogren's syndrome and Behçet's disease developing secondary amyloidosis. Turkiye Klinikleri J Med Sci 2012; 32: 222-225.

4. Sjögren's Syndrome Foundation. SSF launches 5-year breakthrough goal. (Article from The Moisture Seekers January 2012). Available at: http://www.sjogrens.org/ home/about-the-foundation/breakthroughgoal- (accessed January 2018).

5. Hauck T S, Douglas S C, Bookman A A M. Sjogren's syndrome in Canada: diagnosis, treatment and patient perspectives. Connections 2013; 7 (1).

6. Solans-Laqué R, López-Hernandez A, Bosch-Gil J A, Palacios A, Campillo M, Vilardell-Tarres M. Risk, predictors, and clinical characteristics of lymphoma development in primary Sjögren's syndrome. Semin Arthritis Rheum 2011; 41: 415-423.

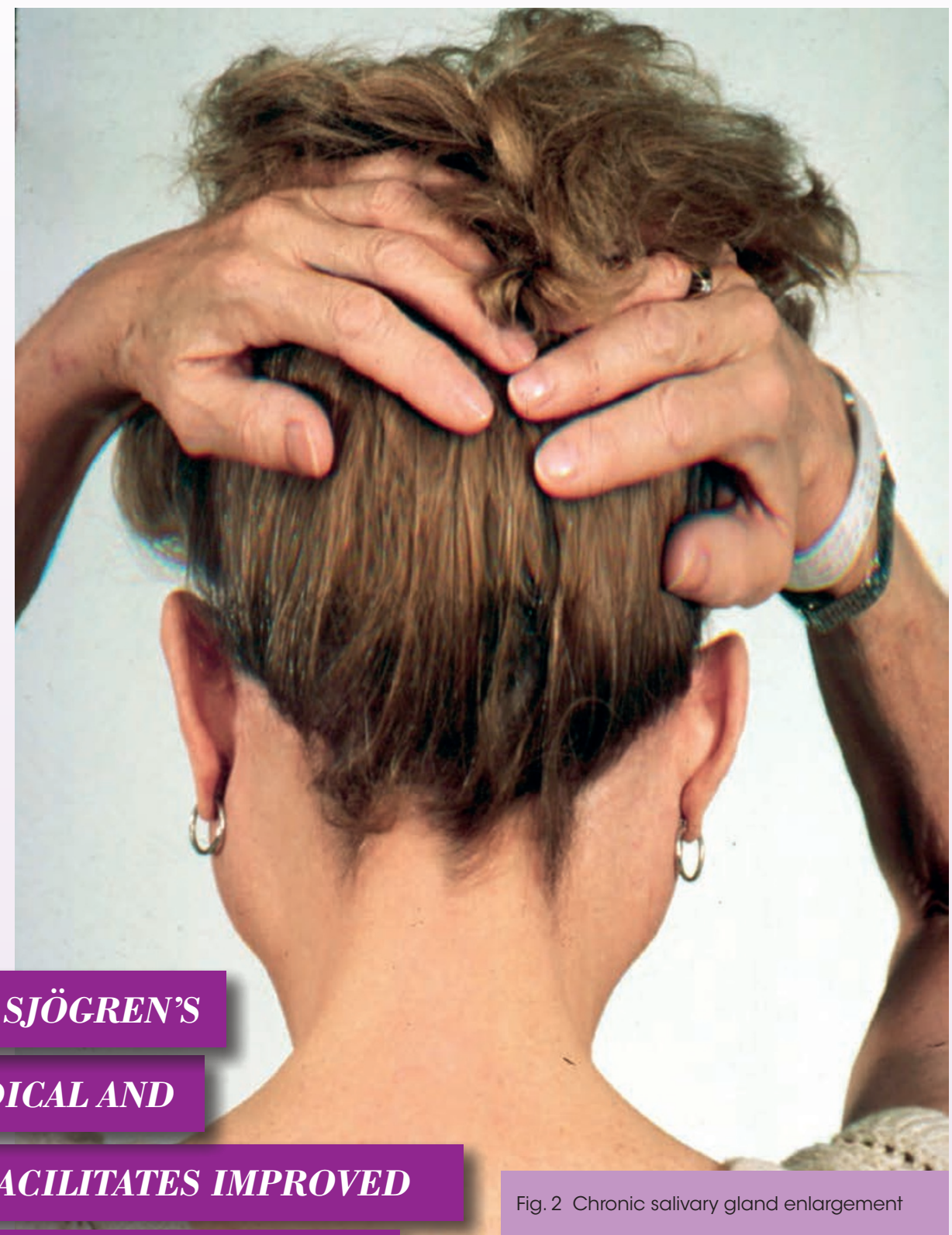

\section{OUTCOMES FOR THE SJÖGREN'S PATIENT'}

7. Grant I A, Hunder G G, Homburger H A, Dyck P J. Peripheral neuropathy associated with sicca complex. Neurology 1997; 48: 855-862.

8. Devins G M. Illness intrusiveness and the psychosocial impact of lifestyle disruptions in chronic life-threatening disease. Adv Ren Replace Ther 1994; 1: 251-263.

9. Bell M, Askari A, Bookman A et al. Sjögren's syndrome: a critical review of clinical management. J Rheumatol 1999; 26: 2051-2061.

10. Felger J C, Lotrich F E. Inflammatory cytokines in depression: neurobiological mechanisms and therapeutic implications. Neuroscience 2013; 246: 199-229.

11. Statistics by Country for Sjogren's syndrome. Extrapolations of prevalence and incidence statistics for Sjogren's syndrome.
Last revision 16 June 2003. Available at: http://www.cureresearch.com/s/sjogrens_ syndrome/stats-country_printer.htm (accessed January 2018).

12. Sjogren's syndrome statistics. From http:// www.sjogrens.org.

13. Shiboski C H, Shiboski S C, Seror R et al. 2016 American College of Rheumatology/ European League Against Rheumatism classification criteria for primary Sjögren's syndrome. Ann Rheum Dis 2017; 76: 9-16.

14. Dry mouth scale launched. Br Dent J 2011; 211: 351 .

15. Papas A, Singh M, Singh M. The effect of a unique Omega-3 supplement on dry mouth and dry eye in Sjögren's patients. Invest Ophthalmol Vis Sci 2007; 48: 377.

16.Sjögren's Syndrome Foundation. Clinical Practice Guideline Resource Sheets.
Available at: http://www.sjogrens.org/ home/research-programs/clinical-practiceguidelines (accessed January 2018).

17.Sjögren's Syndrome Foundation. SSF 5-Year Breakthrough Goal Update: The Average Sjögren's Diagnosis Time Decreases to 3 Years! Available at: http://www.sjogrens.org/home/aboutthe-foundation/breakthrough-goal/5yearupdate (accessed January 2018).

\section{CPD questions}

This article has four CPD questions attached to it which will earn you one hour of verifiable CPD. To access the free BDA CPD hub, go to http:// bit.ly/2e3G0sv 